\title{
Parasites Intestinal: Level of Knowledge and Envisions Mea- sures Mothers of Schoolchildren of Marianao Municipality
}

\author{
Osvaldo Batista Rojas, MSc* \\ Municipal Unit of Hygiene and Epidemiology, Marianao, Cuba \\ *Corresponding author: Osvaldo Batista Rojas, MSc, Assistant Professor and Researcher, Municipal Unit of Hygiene and \\ Epidemiology, Marianao, Havana, Cuba, Tel: +5356790289; +5372652681
}

\begin{abstract}
Objective: To determine the association between the level of knowledge about intestinal parasitosis and preventive measures in mothers of parasitized schoolchildren between the ages of 5 and 11 years of the Marianao municipality in Havana in 2019.

Material and methods: An observational, analytical, crosssectional study that included 195 mothers of schoolchildren between the ages of 5 and 11 years attended in the four Family Medical Offices of Marianao, Havana. Age, marital status, educational level, occupation, level of knowledge about intestinal parasitosis and preventive measures of mothers were recorded.
\end{abstract}

Results: It was obtained that of the surveyed mothers $50.6 \%$ have a regular level of knowledge, $33.3 \%$ obtained a high level of knowledge and $16.1 \%$ obtained a low level of knowledge, according to the preventive measures of the majority of the mothers the majority $(54.4 \%)$ obtained a high level of preventive measures $45.6 \%$ obtained a regular level of preventive measures; Regarding the main research objectives, we find that if there is a significant association between the level of knowledge about intestinal parasitosis and its preventive measures with an OR of $1.9395 \% \mathrm{Cl}$ (1.19-3.13) and a $P$ value of 0.0072 demonstrating that $a$ higher Level of knowledge about intestinal parasitosis leads to a higher level of preventive measures.

Conclusion: According to our results that we obtained in our study after facing the corresponding variables, we can conclude that there is a directly proportional correlation between the level of knowledge about intestinal parasitosis and preventive measures.

\section{Keywords}

Level of knowledge, Prevention measures, Parasitosis, Hygiene

\section{Introduction}

There are several causes of infections of the digestive tract within which are intestinal parasitosis, which develop thanks to the intake of oocysts of protozoa, eggs or larvae of worms, as well as transcutaneously by the penetration of larvae from the ground [1-3].

Improving the level of knowledge about intestinal parasitosis and its preventive measures can be achieved through intervention with educational talks that empower the community, specifically mothers. The presence of protective factors modulates the influence of risk factors, cushioning their impact on children [4,5-9].

Preventive measures that, although they are traditional methods, have resulted and have been chosen as definitive in developed countries as a tool for the prevention and control of intestinal parasitosis, which lie in the use of latrines, drinking water, personal hygiene, footwear, education and especially environmental sanitation.

Based on the above, we ask ourselves the following question: What is the association between the level of knowledge about intestinal parasitosis and its preventive measures of mothers of school children between the ages of 5 and 11 years of the Municipality of Marianao La Habana during the year 2019?

Despite the great advances achieved in antiparasitic therapy and in the methods of diagnosis of parasitic diseases such as the use of molecular biology and genetics, the prevalence rates in some areas of the country are very high, which means that the factors Causes of hyperendemia persist, including the

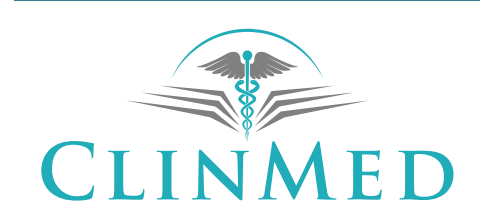

INTERNATIONAL LIBRARY
Citation: Rojas OB (2020) Parasites Intestinal: Level of Knowledge and Envisions Measures Mothers of Schoolchildren of Marianao Municipality. Int J Trop Dis 3:030. doi.org/10.23937/2643-461X/1710030 Accepted: April 22, 2020; Published: April 24, 2020

Copyright: (C) 2020 Rojas OB. This is an open-access article distributed under the terms of the Creative Commons Attribution License, which permits unrestricted use, distribution, and reproduction in any medium, provided the original author and source are credited. 
lack of specific control and prevention programs for this problem [4].

The population mainly affected by this type of disease is childhood, due to immunological immaturity and poor development of hygienic habits leading to negative consequences both physical and intellectual development $[4,5]$.

It has been observed that many mothers lack or have little knowledge about this disease and how to prevent it, therefore, this work on the level of knowledge of intestinal parasitosis and its preventive measures will allow us to have a global vision of the health reality of the mothers.

As a goal we set out to determine the association between the level of knowledge about intestinal parasitosis and preventive measures in mothers of schoolchildren in the Marianao municipality of Havana from 5 to 11 years.

\section{Material and Methods}

An analytical, quantitative, observational, cross-sectional study is carried out to determine the level of knowledge of the mothers of schoolchildren in the Marianao Municipality between the ages of 5 to 11 , representative, viable and of an adequate minimum size. The variables were analyzed: level of knowledge, preventive measures (body hygiene and environmental hygiene), Sociodemographic Factors: Age, School grade, marital status and occupation.

Through the use of a validated survey that meets the necessary parameters to measure the variables established according to the objectives of the present study and having previously requested the corresponding permits from both the authorities of the Ministry of Education and the Ministry of Public Health of the Republic of Cuba.

For quality control of data each of the surveys filled out by mothers will be reviewed schoolchildren ages between 5 to 11 years and not be taken in those that are not properly filled, they are incomplete or are included in the data.

For data collection, mothers of schoolchildren from Abel Santamaria Elementary School were taken whose children ranged from the ages of 5 to 11 years, asking for their support and approval so that they can answer our knowledge level surveys on intestinal parasitosis and other on its preventive measures, this practice was carried out for 4 months.

The method used for the analysis of the data is the analytical statistics oriented to the elaboration and obtaining of the main measures of the study, the statistics of $\mathrm{Chi}^{2}$.

To demonstrate the association of the level of knowledge about intestinal parasitosis and preventive measures, $\mathrm{Chi}^{2}$ and the high vs. regular and low levels of both main variables will be faced in order to interpret them in a better way. The results obtained, after discussion, will confirm or reject the hypotheses raised in the investigation. Then, at the above, the presentation of the results will be presented basically through Table 1 and Figures (Figure 1, Figure 2 and Figure 3).

\section{Results}

We observe that reacting the age and marital status of mothers, single women and with stable performance of groups less than 20 and $21-30$ predominate in the study, a current trend of Cuban society.

It is appreciated that the predominant school level is the pre-university level followed by middle technician the Technician with 75 and 63 respectively, it should be said that our country has been free of illiteracy for 60 years.

In both, it can be seen that the regular level predominates in mothers, with a total of 125 and 105 in Knowledge Level and Preventive Measures respectively. The High Level being the least prevalent.

The association between the level of knowledge about intestinal parasitosis and body hygiene showed, with a p-value of 0.0046 , that mothers with a high knowledge of intestinal parasitosis correspond to a high knowledge of preventive measures, as well as those who have a knowledge Regular or low on intestinal parasitosis also have a regular or low knowledge about their preventive measures, but with a smaller gap between them.

Regarding the association between the level of knowledge about intestinal parasitosis and environmental hygiene where we can find that there is also statistical significance with a p-value of 0.0036 , so that correlation is directly proportional to the level of knowledge thus demonstrating that a high level Knowledge

Table 1: Association of variables chosen in mothers and intestinal parasitism.

\begin{tabular}{|c|c|c|c|c|c|}
\hline $\begin{array}{l}\text { Association between selected variables } \\
\text { and the mother's level of knowledge }\end{array}$ & Height & Regular & Low & Cl $95 \%$ & p value \\
\hline General knowledge about parasitism & 24 & 102 & 69 & $3.25(2.00-3.47)$ & 0.0015 \\
\hline Intestinal parasitosis and body hygiene & 47 & 124 & 24 & $1.99(1.23-3.21)$ & 0.0046 \\
\hline Intestinal parasitosis and environmental hygiene & 29 & 157 & 9 & $2.02(1.26-3.25)$ & 0.0036 \\
\hline Intestinal parasitosis and prophylaxis & 45 & 65 & 85 & $1.93(1.19-3.13)$ & 0.0072 \\
\hline
\end{tabular}

Source: Survey applied. 


\section{Association between age and marital status.}

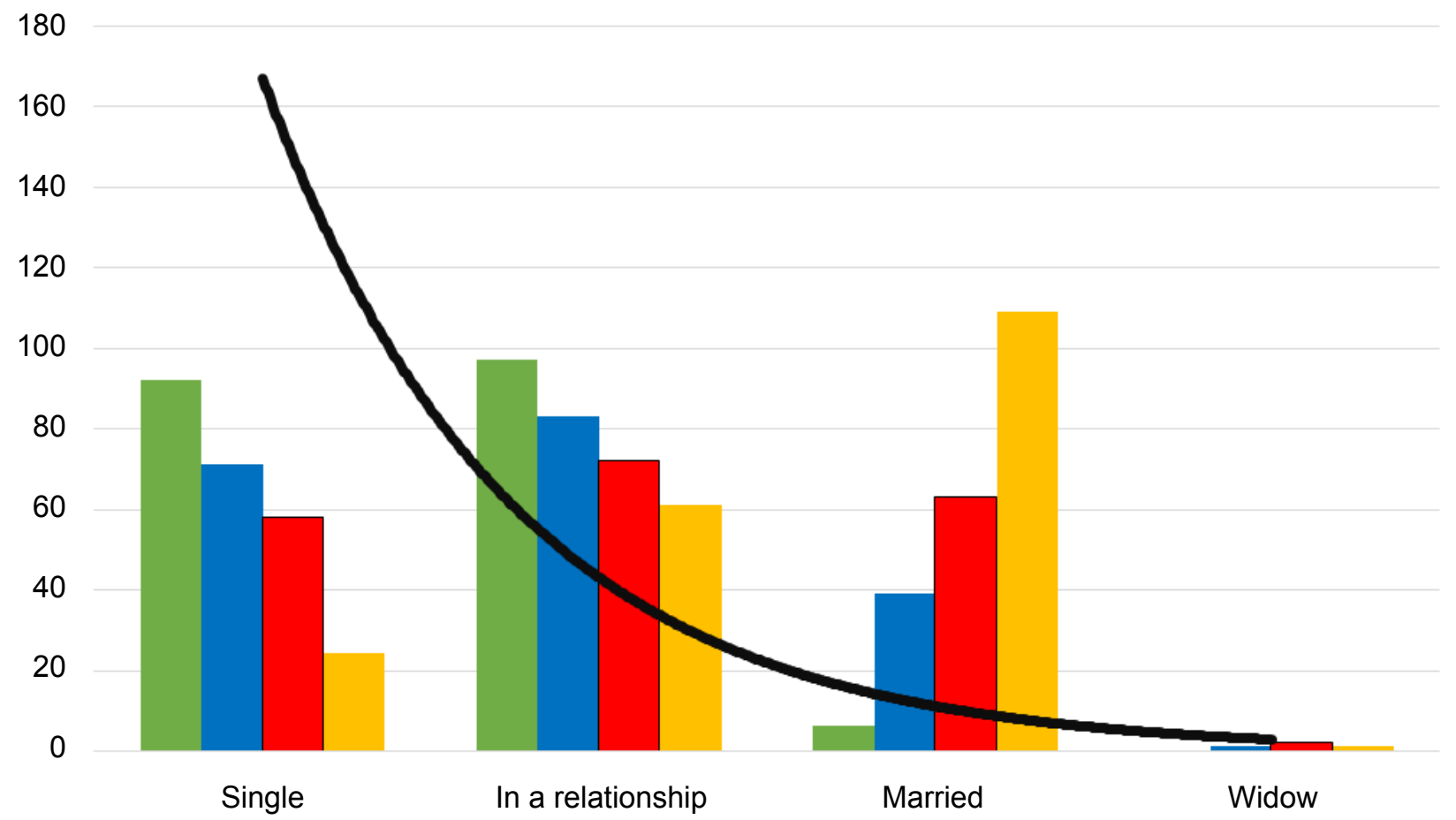

Source: Survey

Figure 1: Relationship between age group and marital status.

\section{Mothers' education levels}

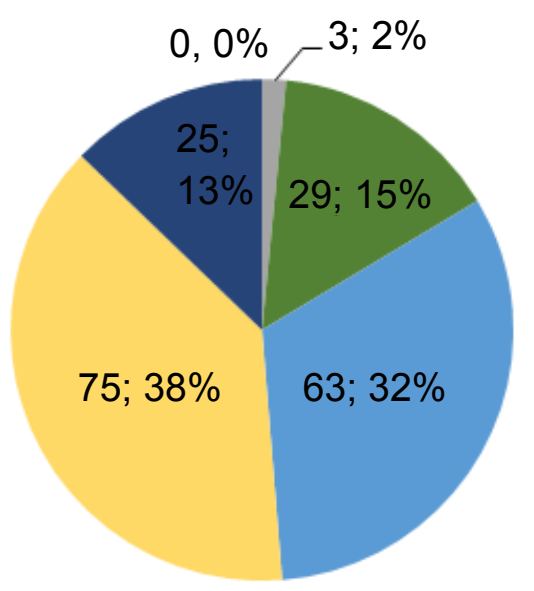

illiterate

Primary Studies

- Hight School

Middle Technician

Preuniversity

- Universitary

Source: Survey

Figure 2: Distribution of levels of educations.

about intestinal parasitosis leads to high preventive measures, as well as regular or low knowledge about intestinal parasitosis leads to regular or low preventive measures.

This together shows the association between the level of knowledge about intestinal parasitosis and its prophylactic measures where a correlation directly proportional to each level is clearly evidenced, demonstrating that a higher level of knowledge about intestinal parasitosis in general leads to greater pre- ventive measures and vice versa at a regular or lower level of knowledge about intestinal parasitosis will lead to having a lower or regular knowledge about preventive measures, this with a $\mathrm{p}$-value of 0.0072 .

\section{Discussion}

Intestinal parasitosis can occur in all people but the child population is more susceptible to it, has a negative impact on health, because children can register a lower weight and are at risk of anemia. Children with parasitosis tend to grow less, are mostly hypoactive and 


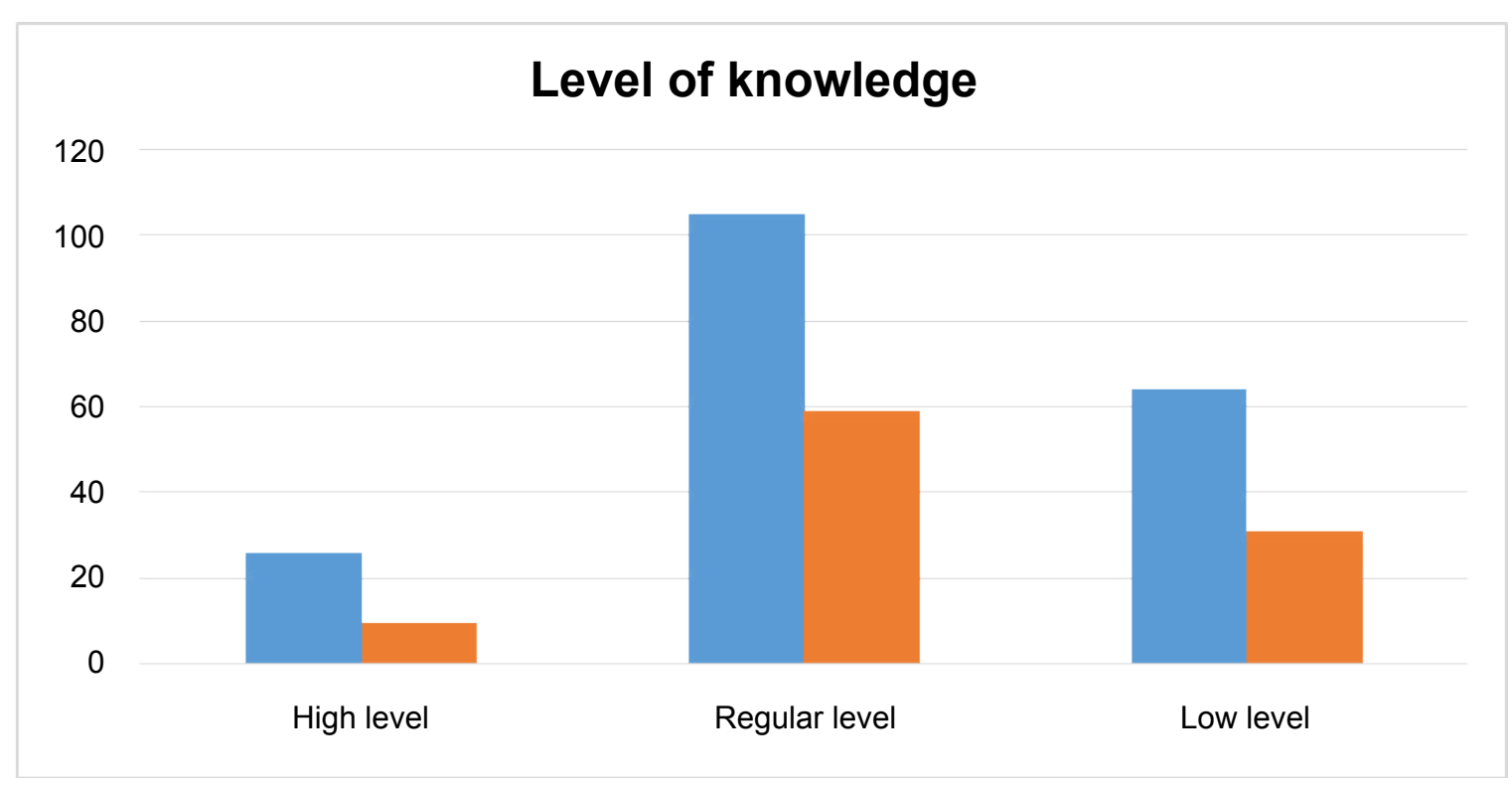

Source: Survey applied

\section{Preventive Measures}

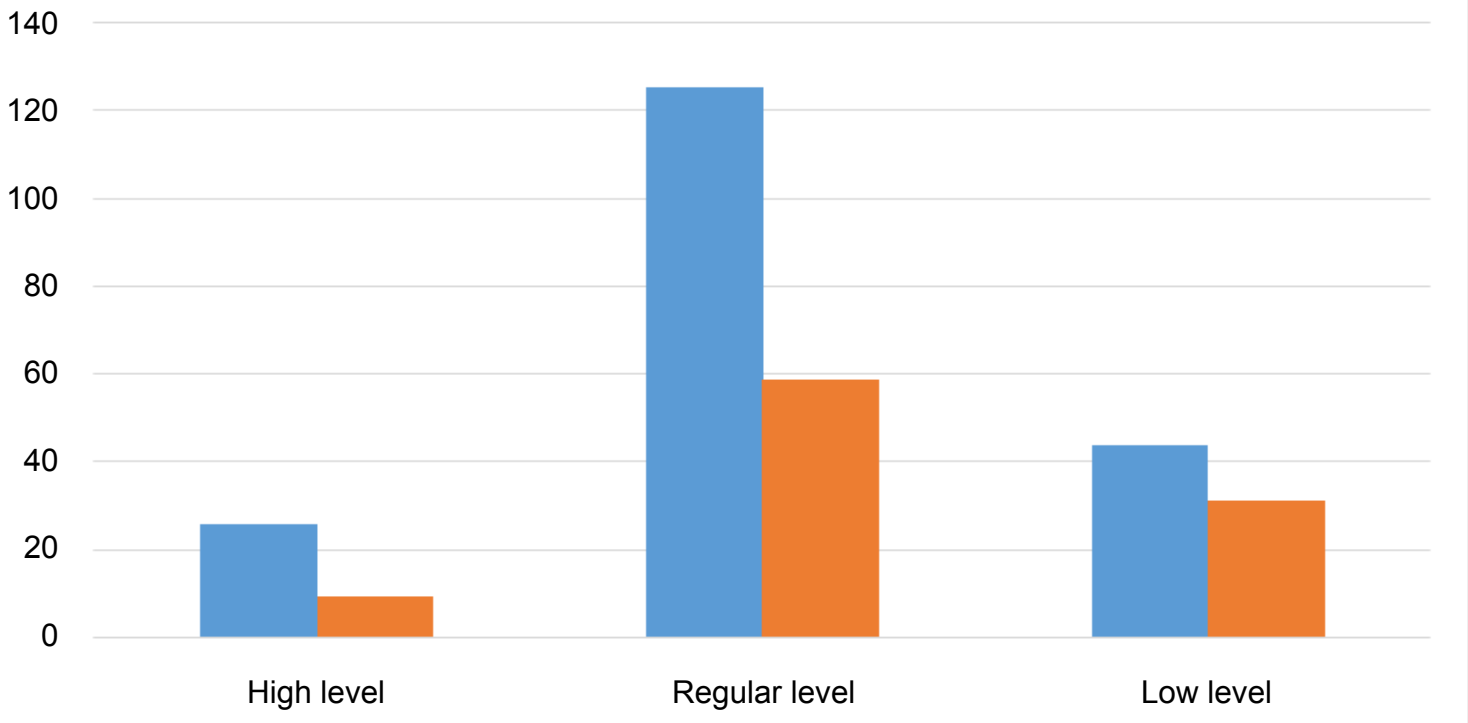

Source: Survey applied

Figure 3: Comparison Chart: A) Distribution of the level of knowledge and B) Preventive measures on intestinal parasitosis. Marianao 2020.

have lower school performance, as parasites affect their physical and intellectual development. So knowledge about this disease as well as the measures for its prevention by the family and the child's environment are essential for better management and control [10-13].

Based on our study, knowledge levels were considered low, regular and high, defining a low level knowledge those who obtained $<6$ points, regular knowledge level those mothers who obtained 6 to 10 points, so the levels Regular and low knowledge indicate that the information that the mother handles about parasitosis is insufficient or insufficient; and those that obtained > 10 points were considered as high level of knowledge, which means that they know enough about the subject, likewise the preventive measures were considered as high, regular and low, defining in turn a low level to those obtained $<12$ points, regulate those who obtained 12 to 22 points and high those who obtained > 22 points, these preventive measures are made up of 3 subconstructs, body hygiene, cavity hygiene and environmental hygiene, which were also stratified in high, regular and low to be able to face them with our variable level of knowledge and thus be able to face together our main variables that are level of knowledge about intestinal parasitosis and preventive measures to see if there is a statistically significant correlation between the two [1-17].

Based on the results obtained, we accept the general alternative hypothesis, which states that there is a significant association of dependence between the 
level of knowledge about intestinal parasitosis and preventive measures in mothers of children between the ages of 5 to 12 years who go to the hospital of vitiate.

In relation to the other sociodemographic variables not contributing to our study objectives, we can observe that the frequency tables on these variables show us that of the total of our sample, the majority of people surveyed are living together, they presented a complete secondary school, they were dedicated to be housewives and ranged between the ages of 18 to 38 years.

\section{Conclusions}

According to our results that we obtained in our study after facing the corresponding variables, we can conclude that there is a directly proportional correlation between the level of knowledge about intestinal parasitosis and preventive measures, which leads us to deduce that the more knowledge about the disease have the mothers, greater preventive measures will be taken in order to significantly reduce the incidence of it.

Body hygiene plays an important role in preventing disease and is significantly related to the level of knowledge about intestinal parasitosis.

Environmental hygiene is one of the most important factors as a preventive measure that is significantly related to the level of knowledge about intestinal parasitosis.

\section{References}

1. Estrada J, Amargós J, Cabrera S, Peña M, Rubio E (2011) Educational strategy for the prevention of parasitism in pediatric ages. Medical Archive Magazine of Camaguey 15: 1-11.

2. Botero D, Parra R, Restrepo GJ, Marcos David Botero MR, Fisher M, et al. (2003) Human parasites. (No. 619: 576.89). World Health Organization.

3. Mandell GL, Bell WEM, William F, Mandell GL, Kourany MMDLS, et al. (2002) Infectious diseases: Principles and practice. Pan American Medical.
4. Jiménez Juan, Vergel Karla, Velásquez García-Sayán Macarena, Vega Fiorella, Uscata Rocío, et al. (2011) Parasitosis in school-age children: Relationship with the degree of nutrition and learning, Lima, Peru. Revista Horizonte Medical 11: 65-69.

5. Aparicio Rodrigo M, Diaz Surgeon Al Intestinal parasitosis (v.1.1/2013). Guide-ABE. Pediatric infections. Quick guide to empirical antimicrobial treatment selection.

6. Claros AM, Peña MM, López MG, Pérez RP, Fontelos PM (2012) Intestinal parasitosis. Diagnostic-therapeutic protocols of the AEP: Pediatric infectology.

7. Rodríguez-Guzmán LM, Hernández-Jerónimo EJ, Rodríguez-García R (2000) Intestinal parasitosis in selected children in an outpatient clinic visit. Rev Mex Pediatr 67: 117-122.

8. Leder K, Weller P, Baron E (2011) Giardiasis: Epidemiology, clinical manifestations, and diagnosis.

9. Leder K, Weller P, Baron E (2004) Enterobiasis (pinworm) and trichuriasis (whipworm).

10. Alarcón M, lannacone J, Blanco YE (2010) Intestinal parasitosis, risk factors, and toxocariasis seroprevalence in residents of the Huaycán industrial park, Lima, Peru. Neotropical Helminthology 4: 17-36.

11. Acha PN, Szyfres B (2003) Zoonoses and communicable diseases common to man and animals. Volume 3, Pan American Health Org.

12. Berenguer JG (2007) Parasitology manual: Morphology and biology of parasites of sanitary interest. Volume 31, Edicions Universitat Barcelona.

13. Virtual Health Library, Descriptors in Health Sciences, terms consultation, Registration number 7574.

14. Botero D, Restrepo M (2003) Intestinal amoebiasis. In: Human parasitosis. $\left(4^{\text {th }}\right.$ edn $)$, Corporation for Biological Research (CIB), Medellín, Colombia, 30-60.

15. Botero D, Restrepo M (2003) Other intestinal protozoosis. In: Human parasitosis. (4 ${ }^{\text {th }}$ edn), Corporation for Biological Research, Medellín, Colombia, 63-91.

16. Mellado Peña MJ (2002) Emerging pathology in pediatric infectious diseases. An Esp Pediatr 56: 423-429.

17. Alvarado L, Romero S (2013) Level of knowledge and practice of conduct promoters in teachers of initial level for prevention of intestinal parasitosis. Florencia de Mora District, (Undergraduate Thesis). Antenor Orrego Private University. 